\title{
Study Of Efficacy Of Dexamethasone As Preemptive Analgesic In Patients Undergoing Total Abdominal Hysterectomy Under Spinal Anaesthesia: A Randomised Controlled Trial
}

\author{
Dr Ravikiran Nikhade ${ }^{1}$, Dr Charuta Gadkari ${ }^{2}$, Dr Prakash Singh ${ }^{3}$, \\ Dr Devashree Warnekar ${ }^{4}$, Dr Anjali Bhure ${ }^{5}$ \\ ${ }^{1}$ Resident, Md Anaesthesiology, Nkpsims, Nagpur \\ ${ }^{2}$ Associate Profesor, Md Anaesthesiology, Nkpsims, Nagpur \\ ${ }^{3}$ Resident, Md Anaesthesiology, Nkpsims, Nagpur \\ ${ }^{4}$ Resident, Md Anaesthesiology, Nkpsims, Nagpur \\ ${ }^{5}$ Head of The Department, Md Anaesthesiology, Nkpsims, Nagpur
}

\begin{abstract}
:
Background: Dexamethasone is an analgesic and anti-emetic agent, that decreases analgesic consumption, yet improve post-operative analgesia.

Objectives: To assess the effect of preemptive Dexamethasone on postoperative analgesia as primary objective. To assess the effect of preemptive Dexamethasone on occurrence of PONV and to study any immediate side effects of Dexamethasone if any as secondary objectives.

Study Design: This is a randomized, double-blind, and placebo-controlled trial conducted over 120 patients undergoing total abdominal hysterectomy under subarachnoid block. Patients were randomized into three groups Group A - Inj. Dexamethasone $8 \mathrm{mg}$ in $10 \mathrm{ml}$ of Normal Saline preemptively + Inj Normal Saline $10 \mathrm{ml}$ IV intraoperatively. Group B-Inj Normal Saline 10ml IV preemptively + Inj. Dexamethasone $8 \mathrm{mg}$ in $10 \mathrm{ml}$ Normal Saline IV intraoperatively. Group C-Inj Normal Saline 10ml IV preemptively + Inj Normal Saline $10 \mathrm{ml} I V$ intraoperatively (control group). Postoperatively, rest and activity pain scores, total analgesic and antiemetic consumption in 24 hours, PONV score, and side effects were recorded.

Results: VAS scores at rest and movement, total analgesic dose required were higher in Groups $B$ and $C$ as compared to group A. Total analgesic dose required were significantly greater in Group $B$ as compared to group A. PONV scores are comparable between group A and group B and significantly lower than control $(C)$. No any immediate side effects observed.

Conclusion: Preemptively Dexamethasone at a dose of $8 \mathrm{mg}$ delays patient request for analgesia and reduces total analgesic consumption postoperatively without any side effects.
\end{abstract}

\section{Introduction}

Pre-emptive analgesia is a treatment that is initiated prior to surgical incision and is operational intraoperatively in order to reduce the physiological consequences of nociceptive transmission provoked by the procedure. Pre-emptive analgesia has the potential to be more effective than a similar analgesic treatment initiated after surgery. Consequently, immediate postoperative pain may be reduced and the development of chronic pain may be prevented. ${ }^{(1)}$ This effect is achieved by suppressing, either together or separately, central or peripheral sensitization. Preemptive analgesia gives rise to a subsiding pain pattern, a decrease in analgesic requirements, and a decline in morbidity, promoting wellness. ${ }^{(2)(3)}$

Initial researches on preemptive analgesia used only two groups for comparison i.e. preoperative intervention group and control group. However, according to newer concept, to prove preemptive effect of any intervention, three groups should be compared i.e. preincisional, postincisional and control.

Based on this newer model of study design, our study is aimed to evaluate primarily the efficacy of preemptive Dexamethasone for postoperative analgesia in patients undergoing total abdominal hysterectomy under spinal anaesthesia. As secondary objectives, we evaluated the effect of preemptive Dexamethasone on PONV and occurrence of immediate side effects if any.

\section{Methods}

After approval of the Institutional Ethics Committee and written informed consent of patients, 120 female patients were enrolled. Inclusion criteria were age between $35 \& 65 \mathrm{yrs}$, weight between $40 \& 70 \mathrm{~kg}$ and patients belonging to ASA class I or II. The patients were excluded if they had psychiatric, neurological disease, diabetes, used regular medication with known or suspected sedative or analgesic effects.

We divided the patients in three groups as follows: 
Group A - Inj. Dexamethasone $8 \mathrm{mg}$ in $10 \mathrm{ml}$ of Normal Saline IV preemptively (1 hour prior to surgery) + Inj Normal Saline $10 \mathrm{ml}$ IV intraoperatively (during closure of vault).

Group B- $\quad$ Inj Normal Saline 10ml IV preemptively + Inj. Dexamethasone $8 \mathrm{mg}$ in $10 \mathrm{ml}$ Normal Saline IV intraoperatively.

Group C- Inj Normal Saline 10ml IV preemptively + Inj Normal Saline 10ml IV intraoperatively (control group).

All the injections were prepared and administered by a person according to group allocation which was done with the help of computer generated table. Another investigator, unaware of group allocation did the intraoperative management of the patients and postoperative monitoring and assessment was done by a different investigator who was again completely blinded to the group allocation.

Pre-anaesthetic assessment was done in participated subjects. All relevant reports on investigation were reviewed. Patients were familiarized with the use of $10 \mathrm{~cm}$ VAS scale.(Fig 1)

Fasting and concurrent medication schedule were followed. In preoperative holding area, IV line was secured with a $20 \mathrm{G}$ canula and drug administration was done according to group allocation.

In the operating room peri-operative monitoring was started with three lead ECG in Std. lead II, non invasive blood pressure (NIBP), pulse oximetry. Lactated Ringer's solution $10 \mathrm{mlkg}^{-1}$ was administered for preloading and subsequently intraoperative hydration was continued as per the calculation, considering abdominal hysterectomy as surgery with moderate tissue trauma, with the help of isotonic crystalloids. Inj Ranitidine $50 \mathrm{mg}$ and Inj Ondansetron $4 \mathrm{mg}$ IV were given as premedication.

Under all aseptic precautions, a midline lumbar puncture was performed with 23 gauge Quincke needle at L3/4 interspace with patients in sitting position and $3.6 \mathrm{ml}$ of hyperbaric Bupivacaine $0.5 \%$ was injected after confirming free flow of clear CSF. Patients were made to lie supine immediately on the OT table without any tilt. Surgeons were allowed to start the surgery after confirming sensory blockade level of T6. Patients were administered Inj Midazolam in incremental doses up to $0.03 \mathrm{mg} \mathrm{kg}^{-1}$ to allay anxiety and received oxygen at the rate of $4 \mathrm{~L}$ per minute via a face mask throughout the procedure.

Those patients who did not attain the T6 sensory blockade were supplemented with Inj Fentanyl 2 $\mathrm{mcg} / \mathrm{kg}$ in titrated doses or received GA with standard institutional protocol. If the surgery lasted for time beyond the action of spinal anaesthesia, similar supplementation was done. These patients were labeled as failure cases due to violation of study protocol.

Hypotension (MAP $\leq 25 \%$ from baseline or systolic pressure $<90 \mathrm{~mm}$ of $\mathrm{Hg}$ ) was treated with Inj Ephedrine $6 \mathrm{mg}$ IV. Bradycardia (HR $<25 \%$ from baseline or HR $<50$ beats/min) was treated with Inj. Atropine $0.6 \mathrm{mg} \mathrm{IV}$.

After surgery, all patients were transferred to PACU. Patients were assessed for intensity of pain with VAS scores at rest and VAS scores on movement at $0^{\text {th }}, 2^{\text {nd }}, 4^{\text {th }}$ and $6^{\text {th }}$ hour in recovery room followed by the intervals at $8^{\text {th }}, 10^{\text {th }}, 16^{\text {th }}$ and $24^{\text {th }}$ hours in ward. Inj Diclofenac Na $75 \mathrm{mg}$ IM was administered as rescue analgesic if VAS score reached $\geq 3$ or on request by patient whichever was earlier. Total analgesic doses given within the first 24-hours postoperatively were recorded.

Scoring of the incidence of nausea and vomiting was done in a manner similar to Belville et $\mathrm{al}^{(5)}$. Episodes of nausea and vomiting were recorded in first 24 hours.(Fig 2a, 2b)

Inj Ondansetron $4 \mathrm{mg}$ was administered intravenously for PONV upon score $\geq 1$. Total number of doses of Inj Ondansetron in 24 hours post operatively were recorded. Patients were assessed regarding occurrence of immediate side effects like stomach upset, skin manifestations (such as rash or itching) as Yes/No survey.

VAS score at rest and on movement as the parameters to reflect difference in VAS amongst the groups with $95 \%$ confidence interval level and $80 \%$ power using one-way ANOVA, the sample size was calculated to be 37 per group. We included 40 patients in each group to compensate for failures of protocol violation.

The data on demographic parameters was collected for each patient in three study groups, and the distributions were obtained. VAS scores at rest and at movement were compared across groups using KruskalWallis test. The paired group comparison of VAS scores was done using Wilcoxon rank sum test. The frequencies of rescue analgesia given to patients in three groups at each time interval were compared using Pearson's Chi-square test. The number of total analgesic dose requirement in three groups was compared using Chi-square test. The Belville et al. score was compared using Fisher's exact test. All the analyses were performed using SPSS 18.0 (SPSS Inc.) and the statistical significance was tested at 5\% level.

\section{Results}

As per the sample size, 40 patients were included in each group but 36 patients in group A, 34 patients in group B and 34 patients in group C could be analyzed. Rest of the patients were excluded from the statistical analysis due to protocol violation. The demographic data and duration of surgery of the 104 patients included in the study were comparable. (Table 1) 
The comparative analysis of VAS scores amongst all the three groups showed that the VAS scores in group A and group B were lower than group C during the entire evaluation period but these could not achieve statistical significance postoperatively at all the time intervals.

VAS scores at rest showed that at $0^{\text {th }}, 6^{\text {th }}$ and $24^{\text {th }}$ hour the difference across three groups was significant with least pain score in group A as compared to group B and C (Table 2).

VAS scores on movement showed that, at $6^{\text {th }}$ and $24^{\text {th }}$ hour the difference across three groups was significant with least pain score in group A as compared to group B and C (Table 3).

The time to first recsue analgesic in group $\mathrm{A}$ is prolonged as compared to group $\mathrm{B}$ and $\mathrm{C}$ and the difference is statistically significent with $\mathrm{p}=0.0042$ (Table 4).

The total number of analgesic doses required in 24 hours. In group A, 26 (76.47\%) patients required 2 doses, while 8 (23.53\%) required 3 doses. In group B, 17 (47.22\%) cases required 2 doses, while 19 (52.78\%) required 3 doses. In group $\mathrm{C}$, all $36(100 \%)$ cases required 3 doses. The total dose requirement across groups was compared for statistical relevance, which resulted into a p-value $<0.0001$ (Fig 3).

The difference in Belville et al. score across three groups was significant at $6 \mathrm{hr}$ and $8 \mathrm{hrs}$ as indicated by p-values 0.001 and 0.0396 respectively (Table 5).

Total anti-emetic dose required in three treatment groups is shown in table - 6 . In group A, only one patient required rescue antiemetic dose, while in group B there were $3(8.33 \%)$ cases who required the rescue antiemetic doses. In group C, the proportion of patients requiring the dose was maximum i.e. 17 (47.23\%). Overall, the difference in the proportion of cases requiring the anti-emetic dose was significantly different across groups as indicated by p-value $<0.0001$. The difference between group A and B is not significant.

\section{Discussion}

Despite improvements in perioperative care, major surgical operations are still followed by postoperative pain hence postoperative pain control is a challenging task for an anaesthesiologist.

Patients are benefitted if pain management is started prior to pain initiation. This requisite can be achieved with preemptive analgesia. It reduces the processing of central sensorial changes and it is an ideal method to reduce the incidence of pain resulting from post-surgery hyperalgesia. ${ }^{(6)(7)(8)}$.

The methods and agents for which preemptive analgesic effectiveness has been researched are mostly NSAIDs $^{(9)}$, epidural analgesia ${ }^{(10)}$, opioids ${ }^{(10)}$, Ketamine ${ }^{(11)}$ and peripheral Local Anaesthetics ${ }^{(12)}$. Glucocorticoids have well-known anti-inflammatory and anti-emetic properties. ${ }^{(13)}$ A single dose of Dexamethasone was not associated with increase incidence of wound infection, it has a low carbohydrate potency (minimally stimulation of glucose formation), its sodium retaining potency is low and allergic/anaphylactic reaction is rare. $^{(14)(15)}$

Dexamethasone with documented analgesic and antiemetic effects has been widely used in various perioperative settings. However, whether incorporation of preemptive single, low-dose Dexamethasone in a current multimodal regimen to manage pain after abdominal hysterectomy provides additional clinical benefit remains unclear. There is a lot of literature discussing preoperative or intraoperative administration of intravenous Dexamethasone claiming its preemptive analgesic effects, however, most of the studies used only two groups i.e control and perioperative administration of Dexamethasone. A positive result in studies designed to show that an analgesic intervention made before surgery is more effective than no intervention at all may suggest a worthwhile clinical benefit. However these results are not evidence for or against a preemptive effect, since such evidence requires a control of the same intervention made at some point after initiation of the surgical procedures ${ }^{(16)}$.

Hence in our study we have divided the patients in three groups - control, preemptive and intraoperative as follows (Fig 4) :

The dose of Dexamethasone was decided based on meta analysis by De Oliveira, Jr. et al. ${ }^{(17)}$ They included 24 trials evaluating the analgesic effects of Dexamethasone perioperatively in three doses as, low dose (less than $0.1 \mathrm{mg} / \mathrm{kg})$, intermediate dose $(0.11-0.2 \mathrm{mg} / \mathrm{kg})$ and high dose $(0.21 \mathrm{mg} / \mathrm{kg})$. They concluded that intermediate dose (between $0.1-2 \mathrm{mg} / \mathrm{kg}$ ) and high dose (>2mg/kg) of Dexamethasone have comparable opioid sparing effect. In our studies we have used $8 \mathrm{mg}$ i.e. intermediate dose of Dexamethasone which is considered to be supraphysiologic dose. They also suggested that preoperative administration of Dexamethasone appears to produce a more consistent analgesic effect compared with intraoperative administration. ${ }^{(17)}$

In our study pain assessment was done by $10 \mathrm{~cm}$ Visual Analog Scale (VAS). If we compare VAS scores at rest across groups over 24 hours, they remained comparable except at $0^{\text {th }}, 4^{\text {th }}, 6^{\text {th }}$, and $24^{\text {th }}$ hour however it is to be noted that VAS scores in group B and C remained so with higher requirement of rescue analgesia. The plasma half life of Dexamethasone is only about 6 hours $^{(18)}$ suggesting a persistent drug effect unrelated to plasma concentration.

Our results are similar with the results of studies by Thangaswamy $\mathrm{C}$ at al ${ }^{(18)}$, Ahmadreza $\mathrm{M}$ et al ${ }^{(19)}$, Jose at $\mathrm{al}^{(20)}$. 
Becky et $\mathrm{al}^{(21)}$ studied the preemptive Effect of Dexamethasone $\left(0.5 \mathrm{mg} \mathrm{kg}^{-1}\right)$ on post-operative opioid requirement in patients who underwent gynecological surgeries. Patients were followed for 12 hours post-operatively for pain intensity at rest and with movement. Pain intensity was significantly lower only after 9 hours in postoperative period in Dexamethasone group.

Hval Kjetil et $\mathrm{al}^{(22)}$ evaluated the effects of adding Dexamethasone to a multimodal postoperative analgesic regimen, including a long-acting nonsteroidal antiinflammatory drug in breast surgery divided into two groups, one with NSAID (Rofecoxib) and other with combination of NSAID (Rofecoxib) and Dexamethasone. They stated that compared with other analgesics, the onset of analgesic effect of Dexamethasone seems to be delayed. They observed analgesic effect of preoperative administration of Dexamethasone only after four hours of postoperative period and it remained persistent till 72 hours. They administered Dexamethasone after induction, before incision. We have given preemptive Dexamethasone 1 hour prior to surgery. We got lower VAS scores at $0^{\text {th }}, 6^{\text {th }}$ and $24^{\text {th }}$ hour.

As stated previously these studies are based on only two groups of patients i.e. preemptive Dexamethasone versus Control, they do not have a group with intraoperative administration of Dexamethasone.

The assessment of VAS scores on movement was done by turning the patient in bed. The VAS scores on movement across the groups throughout the study period were comparable except at $6^{\text {th }}$ and $24^{\text {th }}$ hours which are significant with p-value $<0.0001$ in favor of group A, but most of these readings are taken when the rescue analgesia had been already administered and VAS scores thereafter is a composite effect of basic intervention and rescue analgesic together.

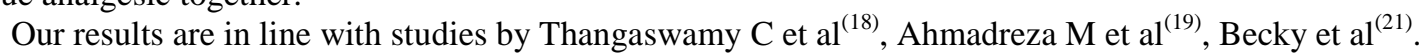

This is consistent with the fact that Dexamethasone has anti-inflammatory potency, with peak antiinflammatory effect at 12-24 hours and duration of action of 36-54 hours. ${ }^{(23)(24)}$

Difference in both, VAS scores at rest and on movement, at 24 th hour has reached statistical significance in favor of preemptive Dexamethasone in our study.

Patients in all three groups required first rescue analgesia within 6 hours postoperatively. In group B and $\mathrm{C}$, all $100 \%$ cases required the first rescue analgesia in first $4 \mathrm{hrs}$. The proportion of cases requiring first rescue analgesia up to $4 \mathrm{hrs}$ in group A was $85.3 \%$ however $14.71 \%$ patients required first rescue analgesia in 4 $6 \mathrm{hrs}$. The requirement of first rescue analgesic has been delayed in these $14.71 \%$ cases in group A and when compared statistically, it is significant with p-value of 0.0042 .

The total number of analgesic doses required in 24 hours was assessed. In group A, 26 (76.47\%) patients required 2 doses, while $8(23.53 \%)$ required 3 doses. In group B, $17(47.22 \%)$ cases required 2 doses, while $19(52.78 \%)$ required 3 doses. In group C, all $36(100 \%)$ cases required 3 doses. The total dose requirement across groups was compared for statistical relevance, which resulted into a p-value $<0.0001$.

$\mathrm{al}^{(21)}$, Feroci et $\mathrm{al}^{(26)}$

Our result match with $\underline{\text { Aissaoui } \mathrm{Y}}$ et $\mathrm{al}^{(25)}$,Thangaswamy $\mathrm{C}$ at $\mathrm{al}^{(18)}$, Ahmadreza $\mathrm{M}$ et $\mathrm{al}^{(19)}$, Becky et

There are studies by José at $\mathrm{al}^{(20)}$, Jun Koh et al ${ }^{(27)}$ where they evaluated the timing of first rescue analgesic and total analgesic requirement for longer duration postoperatively. Their results during first 24 hours match with our results. The meta-analysis by De Oliveria et $\mathrm{al}^{(17)}$ and $\mathrm{N}$. Waldron et al ${ }^{(28)}$ also support our findings.

Mathisen et al ${ }^{(29)}$ studied the effect of preoperative administration of Pregabalin and Dexamethasone in combination with Paracetamol for postoperative pain control after abdominal hysterectomy on one hundred and sixteen patients randomly assigned to either group A (Paracetamol + placebo), group B (Paracetamol + Pregabalin + placebo) or group C (Paracetamol + Pregabalin + Dexamethasone). The 24-h Morphine consumption and pain score, both at rest and during mobilization, were not significantly different between treatment groups. These results are different from our results.

\section{Assessment of PONV}

Assessment of PONV was done by comparing Belville et al. scores across the groups. Scores in group A were compared with group B at similar time points and were found comparable. This suggests that treatment with preoperative administration of Dexamethasone is equally effective compared with intraoperative administration for prophylaxis of PONV. Both the treatment groups had lower Belville et al scores compared to control group at all time points however the difference achieved statistical significance only at $6^{\text {th }}$ and $8^{\text {th }}$ hour. This is consistent with the predominantly anti-nausea effect of Dexamethasone, which has a longer duration of action, as long as 72 hours. ${ }^{(27)}$

The total number of antiemetic doses required in first 24 hours across groups were compared for statistical relevance which resulted into a p-value $<0.0001$. Group A and group B have required significantly less number of rescue antiemetic doses (Inj Ondensetron) as compared to control (group C) however the number of antiemetics required in group A and group B were comparable. There appears to be no added advantage of preoperative administration of Dexamethasone over intraoperative administration with respect to PONV. 
Our results are in line with studies by Thangaswamy $\mathrm{C}$ et $\mathrm{al}^{(18)}$, Jun Koh et al ${ }^{(27)}$, Feroci et al ${ }^{(26)}$, Mathisen et $\mathrm{al}^{(29)}$, Hermans et $\mathrm{al}^{(30)}$.

We monitored patients throughout study period with respect to pulse rate, mean arterial pressure and $\mathrm{SpO}_{2}$. Our patients remained stable clinically. We did not observe any immediate side-effects due to administration of Dxamethasone.

There are a few possible limitations to our study. We could have followed up the patients for extended length of period (up to 72 hours), instead of restricting the evaluation period to 24 hours considering the longer action of Dexamethasone. Steroids are known to raise the blood sugar levels. We did not monitor the blood sugar levels postoperatively however literature mentions that a single dose of Dexamethasone does not affect the blood sugar levels of the body. ${ }^{(14)(31)}$ We could have done blood sugar assessments to confirm the fact.

\section{References}

[1]. Jørgen B. Dahl and Steen Møiniche. Pre-emptive analgesia. Anaesth \& Analg March 2005;100(3): 754-756.

[2]. Alban Latremoliere and Clifford J. Woolf. Central Sensitization: A Generator of Pain Hypersensitivity by Central Neural Plasticity. J Pain September 2009; 10(9): 895-926.

[3]. Woolf CJ, Salter MW. Neuronal plasticity: Increasing the gain in pain. Science 2000;288:1765-1769.

[4]. Carrie L Hicksa, Carl L von Baeyer,The Faces Pain Scale - Revised: toward a common metric in pediatric pain measurement. Pain 2001;93:173-183.

[5]. Bellville JW, Bross IDJ, Howland WS. A method for the clinical evaluation of antiemetic agents. Anesthesiology 1959; 20: 753760 .

[6]. Chiu WK, Cheung LK. Efficacy of preoperative oral rofecoxib in pain control for third molar surgery. Oral Surg Oral Med Oral Pathol Oral Radiol Endod 2005;99(6):47-53

[7]. Joshi A, Parara E, Macfarlane TV. A double-blind randomized controlled clinical trial of the effect of preoperative ibuprofen, diclofenac, paracetamol with codeine and placebo tablets for relief of postoperative pain after removal of impacted third molars. Br J Oral Maxillofac Surg 2004;42(4):299-306.

[8]. Lima PVP, Fontanella V. Analgesic efficacy of Aceclofenac after surgical extraction of impacted lower third molars. Int J Oral Maxillofac Surg 2006;35(6):518-21.

[9]. Semih ARICI, Alp GURBET, Gürkan TÜRKER. Preemptive analgesic effects of intravenous paracetamol in total abdominal hysterectomy. AĞRI 2009;21(2):54-61.

[10]. Ong, Cliff, Lirk, Philipp, Seymour, Robin A. The Efficacy of Preemptive Analgesia for Acute Postoperative Pain Management: A Meta-Analysis. Anesth \& Analg $2005 ; 100$ (3): 757-773.

[11]. Fu, Eugene S, Miguel, Rafael, Scharf, John E. Preemptive Ketamine Decreases Postoperative Narcotic Requirements in Patients Undergoing Abdominal Surgery. Anesth \& Analg May 1997; 84(5): 1086-1090.

[12]. Pasqualucci A, de Angelis V, Contardo R, Colò F, Terrosu G, Donini A, Pasetto A, Bresadola F. Preemptive analgesia: intraperitoneal local anesthetic in laparoscopic cholecystectomy. A randomized, double-blind, placebo-controlled study. Anesthesiology 1996; 85(1):11-20.

[13]. Stubhaug A, Romundstad L, Kaasa T, Breivik H. Methylprednisolone and ketorolac rapidly reduce hyperalgesia around a skin burn injury and increase pressure pain thresholds. Acta Anaesthesiol Scand 2007; 51: 1138-46.

[14]. Rubin RP . Adrenocortical Hormones and Drugs Affecting the Adrenal Cortex. Modern Pharmacology with Clinical Applications. 5th Edn, Little, Brown and Company, 1997; p 727-728.

[15]. Stoelting RK, Hillier SC. Hormones as drugs- Pharmacology and Physiology in Anesthetic Practice. 4th ed. Lippincott Williams \& Raven 2006;p 456-476.

[16]. Dahl JB. The status of pre-emptive analgesia. Curr Opin Anaesthesiol 1995; 8: 323-330.

[17]. Gilda` sio S, De Oliveira, Jr. Marcela D. Perioperative Single Dose Systemic Dexamethasone form Postoperative Pain A Metaanalysis of Randomized Controlled Trials. Anesthesiology $2011 ; 115: 575-88$

[18]. Chitra Rajeswari Thangaswamy, Vimi Rewari, Anjan Trikha. Dexamethasone before total laparoscopic hysterectomy: a randomized controlled dose-response study. J Anesth 2010; 24:24-30.

[19]. Ahmadreza Mohtadi, Sholeh Nesioonpour, Amir Salari. The Effect of Single-Dose Administration of Dexamethasone on Postoperative Pain in Patients Undergoing Laparoscopic Cholecystectomy. Anesth Pain Med August 2014; 4(3): e 17872.

[20]. José Leonardo, Simone Waldyr, Antonio Jorge, Anna Carolina, Ratto Tempestini Horliana. Comparative analysis of preemptive analgesic effect of Dexamethasone and Diclofenac following third molar surgery. Braz Oral Re 2013;27(3):266-277.

[21]. Becky Jno-Baptiste, Marinna D Scarlett and Hyacinth E Harding. The Effect of Dexamethasone on Post-operative Opioid Requirement in Patients who Underwent Gynecology Surgery at the University Hospital in Jamaica. J Anesth Clin Res $2014 ; 5: 11$.

[22]. Hval Kjetil, MD Thagaard, K. Sem. The Prolonged Postoperative Analgesic Effect When Dexamethasone Is Added to a Nonsteroidal Antiinflammatory Drug (Rofecoxib) Before Breast Surgery. Anesth Analg 2007;105:481-6.

[23]. Skjelbred P, Lokken P. Postoperative pain and inflammatory reaction reduced by injection of a corticosteroid. A controlled trial in bilateral oral surgery. Eur J Clin Pharmacol 1982;21: 391-6.

[24]. Falkenstein E, Tillmann HC, Christ M, Feuring M, Wehling M. Multiple actions of steroid hormones- A focus on rapid, nongenomic effects. Pharmacol Rev 2000;52:513-56.

[25]. Aissaoui Y. The effect of a single intravascular dose of Dexamethasone on pain after circumcision, a randomized controlled study. Ann Fr Anesth Reanim 2013;32( 2): 98-103.

[26]. Feroci F, Rettori M, Borrelli A, Lenzi E, Ottaviano A, Scatizzi M. Dexamethasone prophylaxis before thyroidectomy to reduce postoperative nausea, pain, and vocal dysfunction: a randomized clinical controlled trial. Head Neck 2011; 33(6):840-6.

[27]. In Jun Koh MD, Chong Bum Chang MD, Jung Ha Lee MD, Young-Tae Jeon MD, Tae Kyun Kim MD. Preemptive Low-dose Dexamethasone Reduces Postoperative Emesis and Pain After TKA: A Randomized Controlled Study. Clin Orthop Relat Res 2013; 471:3010-3020.

[28]. N. H. Waldron, C. A. Jones, T. J. Gan, T. K. Allen and A. S. Habib. Impact of perioperative Dexamethasone on postoperative analgesia and side-effects: systematic review and meta-analysis. BJA feb 2013;110(2): 191-200.

[29]. O. Mathisen. Pregabalin and Dexamethasone in combination with paracetamol for postoperative pain control after abdominal hysterectomy. A randomized clinical trial. Acta Anaesthesiol Scand 2009; 53: 227-235. 
Study Of Efficacy Of Dexamethasone As Preemptive Analgesic In Patients Undergoing Total..

[30]. V. Hermans, F. De Pooter, F. De Groote, S. De Hert and P. Van der Linden. Effect of Dexamethasone on nausea, vomiting, and pain in paediatric tonsillectomy. BJA 2012, 109 (3): 427-31.

[31]. Samarkandi AH, Shaikh MA, Ahmad RA, Alammar AY. Use of Dexamethasone to reduce postoperative vomiting and pain after pediatric tonsillectomy procedures. Saudi Med J 2004; 25: 1636-1639.

Table 1. Demographic data

\begin{tabular}{|l|l|l|l|}
\hline & Group A $(\mathbf{n}=\mathbf{3 4})$ & Group B $(\mathbf{n}=\mathbf{3 6})$ & Group C $(\mathbf{n}=\mathbf{2 7})$ \\
\hline Age $($ year) & $54.529 \pm 8.382$ & $55.333 \pm 7.907$ & $56.750 \pm 6.942$ \\
\hline Weight $(\mathrm{kg})$ & $59.765 \pm 8.507$ & $57.028 \pm 9.207$ & $56.389 \pm 8.313$ \\
\hline ASA I II & $27 / 7$ & $28 / 8$ & $29 / 7$ \\
\hline Duration of surgery & $88.24 \pm 11.070$ & $89.44 \pm 11.819$ & $89.86 \pm 11.861$ \\
\hline
\end{tabular}

Data are given as mean \pm SD or case number (n); ASA: American Society of Anesthesiologists.

Table 2: Descriptive statistics for VAS scores at rest and its comparison at each time point across treatment groups

\begin{tabular}{|l|l|l|l|l|}
\hline \multirow{2}{*}{ Time point ( in hrs) } & \multicolumn{3}{|c|}{ Groups [Mean(Median)] } & \multirow{2}{*}{ P-value* } \\
\cline { 2 - 4 } & $\mathbf{A}(\mathbf{n}=\mathbf{3 4})$ & $\mathbf{B}(\mathbf{n}=\mathbf{3 6})$ & $\mathbf{C}(\mathbf{n}=\mathbf{3 6})$ & \\
\hline 0 & $1.706(2)^{\mathrm{a}}$ & $2.389(3)^{\mathrm{b}}$ & $2.222(3)^{\mathrm{b}}$ & $\mathbf{0 . 0 4 3 2}(\mathbf{S})$ \\
\hline 2 & $2.529(2)$ & $2.361(2)$ & $2.444(2)$ & $0.7098(\mathrm{NS})$ \\
\hline 4 & $2.118(2)^{\mathrm{a}}$ & $1.694(2)^{\mathrm{b}}$ & $2.000(2)^{\mathrm{a}}$ & $\mathbf{0 . 0 0 1 1}(\mathbf{S})$ \\
\hline 6 & $2.176(2)^{\mathrm{a}}$ & $2.444(2)^{\mathrm{a}}$ & $2.611(3)^{\mathrm{b}}$ & $\mathbf{0 . 0 0 3 8}(\mathbf{S})$ \\
\hline 8 & $2.412(2)$ & $2.389(2)$ & $2.361(2)$ & $0.9496(\mathrm{NS})$ \\
\hline 10 & $2.441(2)$ & $2.056(2)$ & $2.111(2)$ & $0.0831(\mathrm{NS})$ \\
\hline 16 & $2.353(2)$ & $2.444(2)$ & $2.667(3)$ & $0.1060(\mathrm{NS})$ \\
\hline 24 & $1.912(2)^{\mathrm{a}}$ & $2.278(2)^{\mathrm{b}}$ & $2.611(3)^{\mathrm{c}}$ & $<\mathbf{0 . 0 0 0 1}(\mathbf{H S})$ \\
\hline
\end{tabular}

*Obtained using Kruskal-Wallis test; S: Significant; NS: Not significant; HS: Highly significant; Same superscripts associated with means indicate insignificant difference between groups at particular time point as determined using Wilcoxon rank sum test.

Table 3: Descriptive statistics for VAS on movement and its comparison at each time point across treatment groups

\begin{tabular}{|l|l|l|l|l|}
\hline \multirow{2}{*}{ Time point ( in hrs) } & \multicolumn{3}{|c|}{ Groups [Mean(Median)] } & \multirow{2}{*}{ P-value* } \\
\cline { 2 - 4 } & $\mathbf{A}(\mathbf{n}=\mathbf{3 4})$ & $\mathbf{B}(\mathbf{n}=\mathbf{3 6})$ & $\mathbf{C}(\mathbf{n}=\mathbf{3 6})$ & \\
\hline 0 & $2.235(2)$ & $3.000(4)$ & $2.778(4)$ & $0.1019(\mathrm{NS})$ \\
\hline 2 & $3.088(2)$ & $3.056(2)$ & $2.861(2)$ & $0.7232(\mathrm{NS})$ \\
\hline 4 & $2.324(2)$ & $2.000(2)$ & $2.000(2)$ & $0.0517(\mathrm{NS})$ \\
\hline 6 & $2.412(2)^{\mathrm{a}}$ & $2.806(2)^{\mathrm{a}}$ & $3.222(4)^{\mathrm{b}}$ & $\mathbf{0 . 0 0 4 4}(\mathbf{S})$ \\
\hline 8 & $2.882(2)$ & $2.833(2)$ & $2.667(2)$ & $0.7305(\mathrm{NS})$ \\
\hline 10 & $2.824(2)$ & $2.528(2)$ & $2.222(2)$ & $0.1262(\mathrm{NS})$ \\
\hline 16 & $2.794(2)$ & $2.861(2)$ & $3.222(4)$ & $0.2116(\mathrm{NS})$ \\
\hline 24 & $\left.2.000(2)^{\mathrm{a}}\right)$ & $2.667(2)^{\mathrm{b}}$ & $3.111(4)^{\mathrm{b}}$ & $<\mathbf{0 . 0 0 0 1}(\mathbf{H S})$ \\
\hline
\end{tabular}

*Obtained using Kruskal-Wallis test; S: Significant; NS: Not significant; HS: Highly significant; Same superscripts associated with means indicate insignificant difference between groups at particular time point as determined using Wilcoxon rank sum test

Table 4 : Time to first rescue analgesic

\begin{tabular}{|l|l|l|l|}
\hline \multirow{2}{*}{ Time in hrs (Post-operative) } & \multicolumn{3}{|l|}{ Group [No. (\%)] } \\
\cline { 2 - 4 } & $\mathbf{A}(\mathbf{n}=\mathbf{3 4})$ & $\mathbf{B}(\mathbf{n}=\mathbf{3 6})$ & $\mathbf{C}(\mathbf{n}=\mathbf{3 6})$ \\
\hline 0 & $14(41.18)$ & $21(58.33)$ & $21(58.33)$ \\
\hline $2-4$ & $15(44.12)$ & $15(41.67)$ & $15(41.67)$ \\
\hline $4-6$ & $5(14.71)$ & 0 & 0 \\
\hline
\end{tabular}

P-value: $<0.0001$ obtained using Pearson's Chi-square test

Table 5: : Descriptive statistics for Belville et al. score and its comparison at each time point across treatment groups

\begin{tabular}{|c|c|c|c|c|c|c|c|c|c|c|}
\hline \multirow[t]{3}{*}{ Time point ( in hrs) } & \multicolumn{9}{|c|}{ Groups / Belville et al. score (Number) } & \multirow[t]{3}{*}{ P-value* } \\
\hline & \multicolumn{3}{|c|}{$\mathbf{A}(\mathbf{n}=34)$} & \multicolumn{3}{|c|}{ B $(\mathbf{n}=36)$} & \multicolumn{3}{|c|}{$\mathrm{C}(\mathrm{n}=\mathbf{3 6})$} & \\
\hline & $\mathbf{0}$ & 2 & 3 & $\mathbf{0}$ & 2 & 3 & $\mathbf{0}$ & 2 & 3 & \\
\hline 0 & 34 & 0 & 0 & 36 & 0 & 0 & 36 & 0 & 0 & - \\
\hline 2 & 33 & 0 & 1 & 35 & 0 & 1 & 36 & 0 & 0 & - \\
\hline 4 & 34 & 0 & 0 & 36 & 0 & 0 & 36 & 0 & 0 & - \\
\hline 6 & 34 & 0 & 0 & 36 & 0 & 0 & 27 & 9 & 0 & $0.001(\mathrm{~S})$ \\
\hline 8 & 34 & 0 & 0 & 33 & 3 & 0 & 30 & 6 & 0 & $0.0396(\mathrm{~S})$ \\
\hline 10 & 34 & 0 & 0 & 36 & 0 & 0 & 35 & 1 & 0 & $0.321(\mathrm{NS})$ \\
\hline 16 & 34 & 0 & 0 & 36 & 0 & 0 & 35 & 1 & 0 & $0.321(\mathrm{NS})$ \\
\hline 24 & 34 & 0 & 0 & 36 & 0 & 0 & 36 & 0 & 0 & \\
\hline
\end{tabular}

*Obtained using Fisher's exact test; S: Significant; NS: Not significant 
Study Of Efficacy Of Dexamethasone As Preemptive Analgesic In Patients Undergoing Total..

Table 6: Counts of total anti-emetic doses required in three treatment groups

\begin{tabular}{|l|l|l|l|}
\hline \multirow{2}{*}{ No.of Doses } & \multicolumn{3}{|l|}{ Groups[No (\%)] } \\
\cline { 2 - 4 } & $\mathbf{A}(\mathbf{n}=\mathbf{3 4 )}$ & $\mathbf{B}(\mathbf{n}=\mathbf{3 6})$ & $\mathbf{C}(\mathbf{n}=\mathbf{3 6})$ \\
\hline 0 & $33(97.06)$ & $33(91.67)$ & $19(52.78)$ \\
\hline 1 & $1(2.94)$ & $3(8.33)$ & $17(47.23)$ \\
\hline
\end{tabular}

P-value: $<0.0001$ obtained using Chi-square test

\section{Figures}

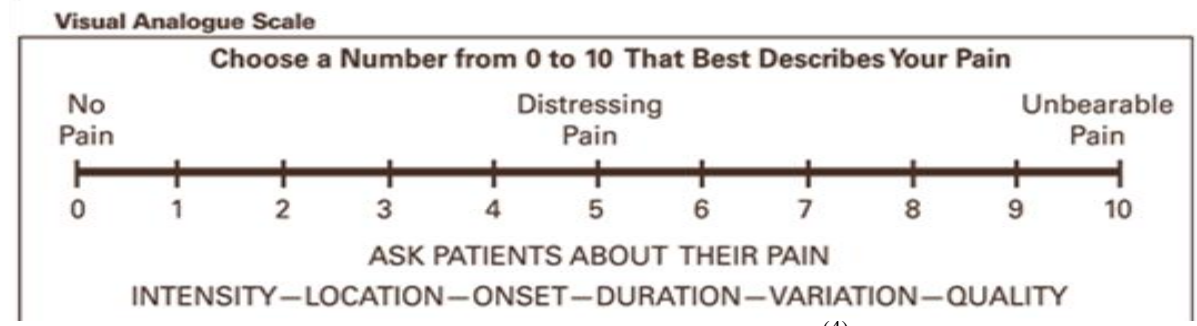

Figure 1: Visual Analogue Scale ${ }^{(4)}$

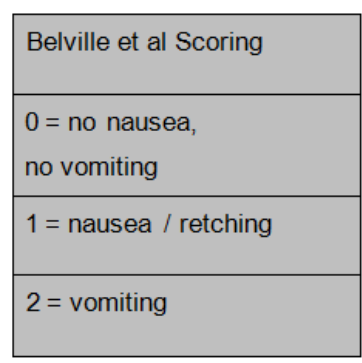

Fig 2a: Belville et al Scoring ${ }^{(5)}$

\begin{tabular}{|l|}
\hline $\begin{array}{l}\text { Nausea - defined as a subjectively } \\
\text { unpleasant sensation associated with the } \\
\text { urge to vomit. }\end{array}$ \\
\hline $\begin{array}{l}\text { Vomiting - defined as the forceful expulsion } \\
\text { of gastric contents. }\end{array}$ \\
\hline $\begin{array}{l}\text { Retching - defined as the laboured, } \\
\text { spasmodic, rhythmic contraction of the } \\
\text { respiratory muscles without expulsion of the } \\
\text { gastric contents. }\end{array}$ \\
\hline
\end{tabular}

Fig 2b : Definition

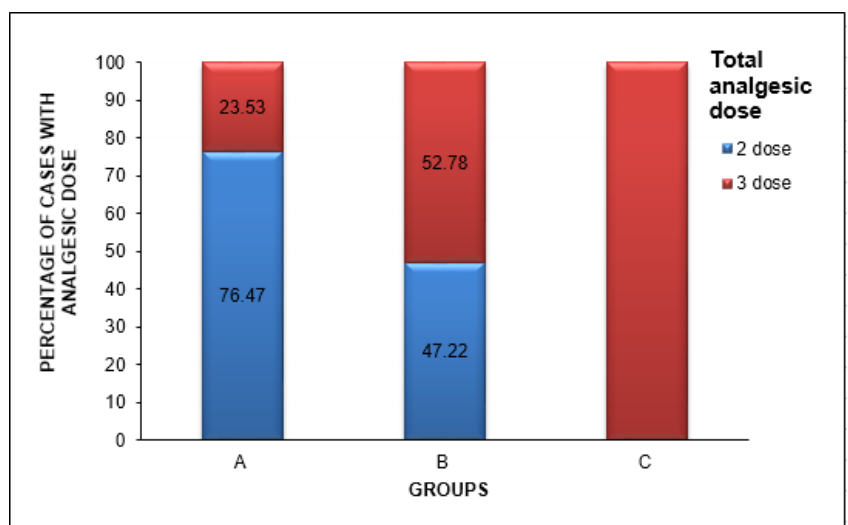

Figure 3: Stacked bar chart showing percentage of cases with analgesic dose in three treatment groups.

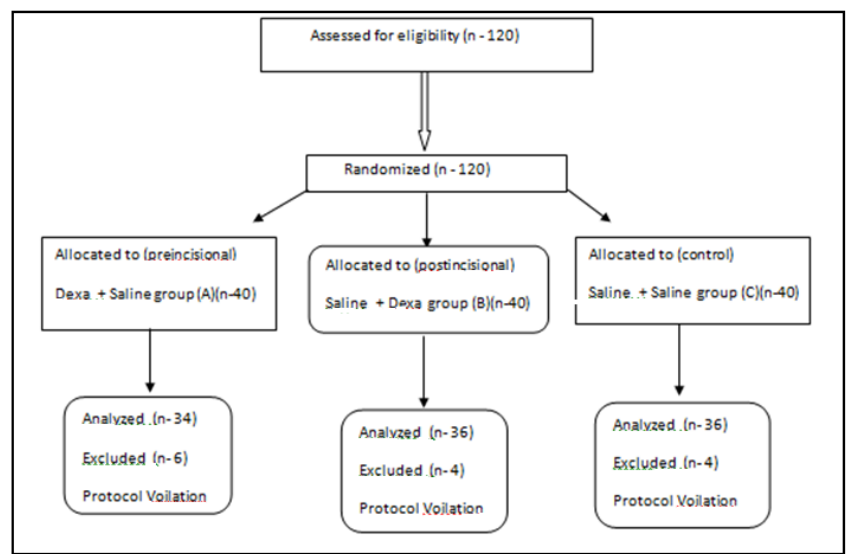

Figure 4: Flow diagram showing the study design. 